TUM-T39-98-20

JYFL-13/98

hep-ph/9808330

June 15, 2021

\title{
Nuclear Quark and Gluon Distributions in Coordinate Space
}

\author{
M. Vänttinen円, G. Piller, L. Mankiewicz and W. Weise \\ Physik Department, Technische Universität München, \\ D-85747 Garching, Germany \\ K. J. Eskola \\ Department of Physics, University of Jyväskylä, \\ P.O.Box 35, FIN-40351 Jyväskylä, Finland
}

\begin{abstract}
In coordinate space, quark and gluon distributions of the nucleon are defined as correlation functions involving two field operators separated by a light-cone distance $y^{+}=2 l$. We study the nuclear modifications of these distributions. The largest effect is a strong depletion of parton distributions (shadowing) at large longitudinal distances, which starts for all parton species at $l=2 \mathrm{fm}$, i.e. at the average nucleonnucleon separation in nuclei. On the other hand, the nuclear radius does not play a significant role. At $l \lesssim 1 \mathrm{fm}$, nuclear modifications of parton distributions are small. The intrinsic structure of individual nucleons is evidently not very much affected by nuclear binding. In particular, there is no evidence for a significant increase of the quark or gluon correlation length in bound nucleons.
\end{abstract}

PACS: 13.60.Hb, 14.20.Dh, 24.85.+p

\footnotetext{
${ }^{\dagger}$ Work supported in part by BMBF

*Alexander von Humboldt fellow
} 


\section{Introduction}

A significant difference between nucleon and nuclear structure functions was first observed in deep inelastic scattering by the EMC collaboration [G]. Since then, considerable experimental and theoretical efforts have been devoted to detailed investigations of nuclear modifications of parton distributions (for a review see e.g. [2]). Their phenomenological discussion has been carried out almost entirely in momentum space. However, interesting insights can be obtained also in coordinate space. Here the parton distributions are defined in leading twist accuracy as correlation functions involving two quark or gluon field operators, separated by a light-cone distance $y^{+}$[3, 团. In deep inelastic scattering from nuclei as viewed in the laboratory frame, where the target is at rest, the longitudinal distance $y^{+} / 2$ involved in the parton correlation functions can be compared with typical length scales provided by the nucleus. This offers new possibilities for extracting information on the nature of nuclear effects in parton distributions [5, 6].

To demonstrate the relevance of coordinate-space distances in nuclear parton distributions we focus on deep inelastic scattering. Consider first the scattering from a free nucleon with momentum $P$ and invariant mass $M$ in the laboratory frame. The momentum transfer $q$, carried by the exchanged virtual photon, is taken in the (longitudinal) $\hat{3}$-direction, $q^{\mu}=\left(\nu, \overrightarrow{0}_{\perp}, \sqrt{\nu^{2}+Q^{2}}\right)$, with $Q^{2}=-q^{2}$. In the Bjorken limit, $\nu^{2} \gg Q^{2} \gg M^{2}$ with $x=Q^{2} /(2 M \nu)$ fixed, the light-cone components of the photon momentum, $q^{ \pm}=q^{0} \pm q^{3}$, are $q^{+} \simeq 2 \nu$ and $q^{-} \simeq-M x$. All information about the response of the target to the high-energy interaction is in the hadronic tensor

$$
W_{\mu \nu}(q, P) \sim \int d^{4} y e^{i q \cdot y}\left\langle P\left|J_{\mu}(y) J_{\nu}(0)\right| P\right\rangle,
$$

defined as the Fourier transform of a product of the electromagnetic currents $J_{\mu}$, with its expectation value taken between the nucleon states. Using

$$
q \cdot y=\frac{1}{2}\left(q^{+} y^{-}+q^{-} y^{+}\right)-\vec{q}_{\perp} \cdot \vec{y}_{\perp}=\nu y^{-}-\frac{M x}{2} y^{+},
$$

one obtains the following coordinate-space resolutions along the coordinates $y^{ \pm}=t \pm y^{3}$ :

$$
\delta y^{-} \sim \frac{1}{\nu} \quad \text { and } \delta y^{+} \sim \frac{1}{M x} .
$$

At $y^{-}=0$ the current correlation function in Eq. (1) is not analytic since it vanishes for $y^{+} y^{-}-\left(\vec{y}_{\perp}\right)^{2}<0$ because of causality (see e.g. [7]). Indeed in perturbation theory it turns out to be singular at $y^{-}=0$. Assuming that the integrand in (11) is an analytic function of $y^{-}$elsewhere, this implies that $W_{\mu \nu}$ is dominated for $q^{+} \rightarrow \infty$ by contributions from $y^{-}=$ 0 . Using causality one then finds that, in the transverse plane, only contributions from $\left(\vec{y}_{\perp}\right)^{2} \simeq 1 / Q^{2}$ are relevant: in deep inelastic scattering the hadronic tensor is dominated by contributions from the light cone, i.e. $y^{2}=0$.

Furthermore, Eq. (3) suggests that along the light cone, one is dominantly probing larger distances as $x$ is decreased. It has been shown that such a behavior is consistent with 


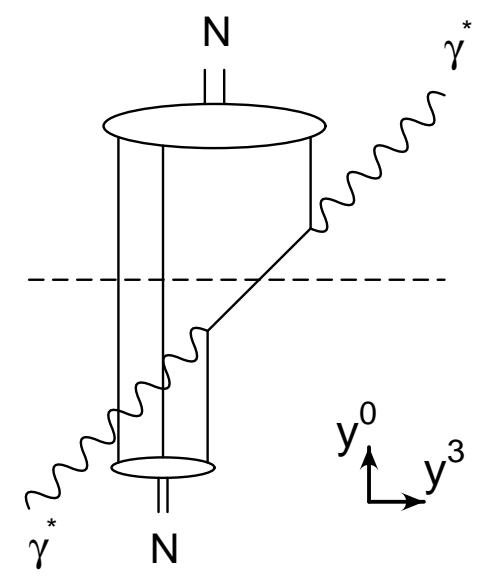

Figure 1: A Feynman diagram from Ref. [6] illustrating the space-time pattern of deep inelastic scattering.

approximate Bjorken scaling [8]. An analysis of nucleon structure functions in coordinate space as carried out in Section 3 confirms the above conjecture. In the Bjorken limit the dominant contributions to the hadronic tensor at small $x$ come from light-like separations of order $y^{+} \sim 1 /(M x)$ between the electromagnetic currents.

In the laboratory frame our considerations imply that deep inelastic scattering involves a longitudinal correlation length

$$
y^{3} \simeq \frac{y^{+}}{2} \equiv l
$$

of the virtual photon. Consequently, large longitudinal distances are important in the scattering process at small $x$. This can also be deduced in the framework of time-ordered perturbation theory (see e.g. [9]), where the typical propagation length of hadronic configurations present in the interacting photon is $y^{3} \sim 1 /(2 M x)$, in accordance with our discussion.

The space-time pattern of deep inelastic scattering is illustrated in Fig. 1 in terms of the imaginary part of the forward Compton amplitude: the virtual photon interacts with a quark or antiquark which is displaced a distance $y^{+}$along the light cone. The characteristic laboratory frame correlation length $l$ is one half of that distance. This behavior is naturally implemented in coordinate-space (or so-called Ioffe-time) distribution functions. They are related to momentum-space distributions through Fourier transformation and thus select contributions to the scattering process which result from specific light-cone distances $y^{+}$. For example, the charge-conjugation-even quark distribution in coordinate space reads:

$$
\mathcal{Q}\left(y^{+}, Q^{2}\right) \equiv \int_{0}^{1} d x\left[q\left(x, Q^{2}\right)+\bar{q}\left(x, Q^{2}\right)\right] \sin \left(\frac{M y^{+}}{2} x\right)
$$

Here $q\left(x, Q^{2}\right)$ and $\bar{q}\left(x, Q^{2}\right)$ are the quark and antiquark momentum-space distribution functions which depend on Bjorken $x$ and on the momentum scale $Q^{2}$. At lowest order 
in the strong coupling constant $\alpha_{s}$, the distribution $\mathcal{Q}$ is related to the structure function $F_{2}$, which is measured in deep inelastic scattering, through: ${ }^{1}$

$$
\mathcal{F}_{2}\left(y^{+}, Q^{2}\right)=\sum_{f} e_{f}^{2} \mathcal{Q}_{f}\left(y^{+}, Q^{2}\right)=\int_{0}^{1} \frac{d x}{x} F_{2}\left(x, Q^{2}\right) \sin \left(\frac{M y^{+}}{2} x\right)
$$

where $e_{f}$ is the fractional electromagnetic charge of a quark with flavor $f$.

If one compares the longitudinal correlation length $l$ from Eq. (4) with the average nucleon-nucleon distance in the nucleus, $d \approx 2 \mathrm{fm}$, one can distinguish two different kinematic regions:

(i) At small distances, $l<d$, the virtual photon scatters incoherently from the individual hadronic constituents of the nuclear target. Possible modifications of $\mathcal{Q}\left(y^{+}\right)$in this region are caused by bulk nuclear effects such as binding and Fermi motion.

(ii) At larger distances, $l>d$, it is likely that several nucleons participate collectively in the interaction. Modifications of $\mathcal{Q}\left(y^{+}\right)$are now expected to come from the coherent scattering of the photon on several nucleons in the target. Using $l \sim 1 /(2 M x)$, this region corresponds to the kinematic domain $x \lesssim 0.05$.

This suggests that the nuclear modifications seen in coordinate-space distributions will be quite different in the regions $l>2 \mathrm{fm}$ and $l<2 \mathrm{fm}$.

In the following we first recall, in Section 2, the definition of coordinate-space distribution functions and review their relation to QCD operators. In Section 3 we discuss the coordinate-space distribution functions of free nucleons. Nuclear modifications of quark and gluon distributions are investigated in Section 4. A short summary is given in Section 5 .

\section{Coordinate-space distribution functions}

It is useful to express coordinate-space distributions in terms of a dimensionless variable. For this purpose let us introduce the light-like vector $n^{\mu}$ with $n^{2}=0$ and $P \cdot n=P^{0}-P^{3}$. As discussed in the introduction, dominant contributions to Eq. (1) come from the vicinity of the light cone, where $y$ is approximately parallel to $n$. The dimensionless variable $z=y \cdot P$ then plays the role of a coordinate conjugate to Bjorken $x$. It is useful to bear in mind that the value $z=5$ corresponds in the laboratory frame to a light-cone distance $y^{+}=2 z / M \approx 2 \mathrm{fm}$ or, equivalently, to a longitudinal distance $l \equiv y^{+} / 2 \approx 1 \mathrm{fm}$.

In accordance with the charge conjugation $(C)$ properties of momentum-space quark and gluon distributions, one defines coordinate-space distributions by [10]

$$
\mathcal{Q}\left(z, Q^{2}\right) \equiv \int_{0}^{1} d x\left[q\left(x, Q^{2}\right)+\bar{q}\left(x, Q^{2}\right)\right] \sin (z x),
$$

\footnotetext{
${ }^{1}$ In higher orders in $\alpha_{s}$ this is a matter of scheme conventions.
} 


$$
\begin{aligned}
\mathcal{Q}_{v}\left(z, Q^{2}\right) & \equiv \int_{0}^{1} d x\left[q\left(x, Q^{2}\right)-\bar{q}\left(x, Q^{2}\right)\right] \cos (z x), \\
\mathcal{G}\left(z, Q^{2}\right) & \equiv \int_{0}^{1} d x x g\left(x, Q^{2}\right) \cos (z x),
\end{aligned}
$$

where $q, \bar{q}$ and $g$ are the momentum-space quark, antiquark and gluon distributions, respectively. Flavor degrees of freedom are suppressed here.

In leading twist accuracy, the coordinate-space distributions (7) 9) are related to forward matrix elements of non-local QCD operators on the light cone [3, 4]:

$$
\begin{aligned}
\mathcal{Q}\left(z, Q^{2}\right) & =\frac{1}{4 i P \cdot n}\langle P|\bar{\psi}(y) \not \Gamma(y) \psi(0)| P\rangle_{Q^{2}}-(y \leftrightarrow-y), \\
\mathcal{Q}_{v}\left(z, Q^{2}\right) & =\frac{1}{4 P \cdot n}\langle P|\bar{\psi}(y) \not h \Gamma(y) \psi(0)| P\rangle_{Q^{2}}+(y \leftrightarrow-y), \\
\mathcal{G}\left(z, Q^{2}\right) & =n^{\mu} n^{\nu} \frac{1}{2(P \cdot n)^{2}}\left\langle P\left|G_{\mu \lambda}(y) \Gamma(y) G_{\nu}^{\lambda}(0)\right| P\right\rangle_{Q^{2}} .
\end{aligned}
$$

Here $\psi$ denotes the quark field and $G_{\mu \nu}$ the gluon field strength tensor. The path-ordered exponential

$$
\Gamma(y)=\mathrm{P} \exp \left[i g y^{\mu} \int_{0}^{1} d \lambda A_{\mu}(\lambda y)\right]
$$

where $g$ denotes the strong coupling constant and $A^{\mu}$ the gluon field, ensures gauge invariance of the parton distributions. Note that an expansion of the right-hand side of Eqs. (7 -9) and (10-12) around $y=0$ leads to the conventional operator product expansion for parton distributions [7].

The functions $\mathcal{Q}(z), \mathcal{Q}_{v}(z)$ and $\mathcal{G}(z)$ characterize the mobility of partons in coordinate space. Consider, for example, the quark distributions $\mathcal{Q}(z)$ and $\mathcal{Q}_{v}(z)$. The matrix elements in (10,11) have an obvious physical interpretation: they measure the overlap between the nucleon ground state and a state in which one quark has been displaced along the light cone from 0 to $y$. Antiquarks undergo this sequence in opposite order.

\section{Parton distribution functions of free nucleons}

In this section we discuss the properties of coordinate-space distribution functions of free nucleons. Examples of the distributions (10 12) using the CTEQ4L parametrization [11] of momentum-space quark and gluon distributions taken at a momentum scale $Q^{2}=4$ $\mathrm{GeV}^{2}$, are shown in Fig. 2.

Some general features can be observed: the $C$-even quark distribution $\mathcal{Q}(z)$ rises at small values of $z$, develops a plateau at $z \gtrsim 5$, and then exhibits a slow rise at very large $z$. At $z \lesssim 5$, the function $z \mathcal{G}(z)$ behaves similarly as $\mathcal{Q}(z)$. For $z \gtrsim 5, z \mathcal{G}(z)$ rises somewhat faster than $\mathcal{Q}(z)$. The $C$-odd (or valence) quark distribution $\mathcal{Q}_{v}(z)$ starts with a finite value at small $z$, then begins to fall at $z \simeq 3$, and vanishes at large $z$. Recall that, in the laboratory frame, the scale $z \simeq 5$ at which a significant change in the behavior of coordinate-space distributions occurs, represents a longitudinal distance comparable to the typical size of a nucleon. 


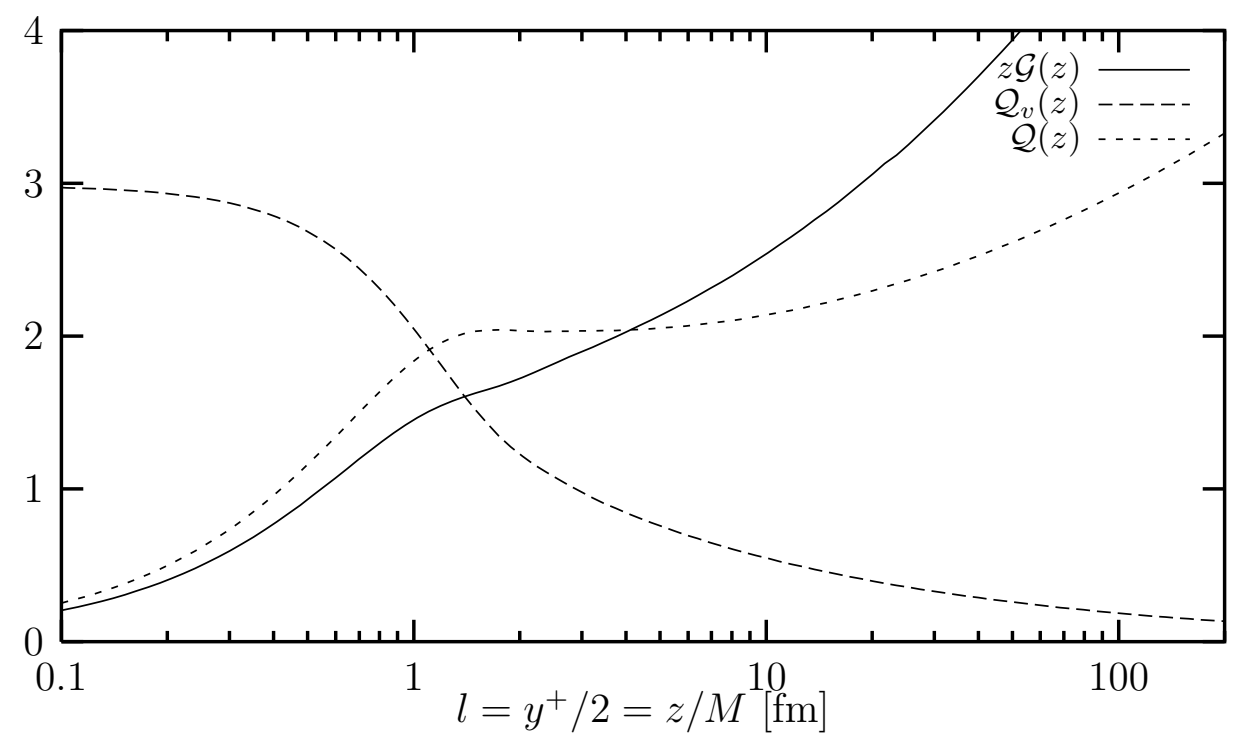

Figure 2: Coordinate-space quark and gluon distributions resulting from the CTEQ4L parametrization of momentum-space distributions, taken at a momentum transfer $Q^{2}=4$ $\mathrm{GeV}^{2}$. A sum over the $u$ and $d$ quarks is implied in the functions $\mathcal{Q}_{v}$ and $\mathcal{Q}$.

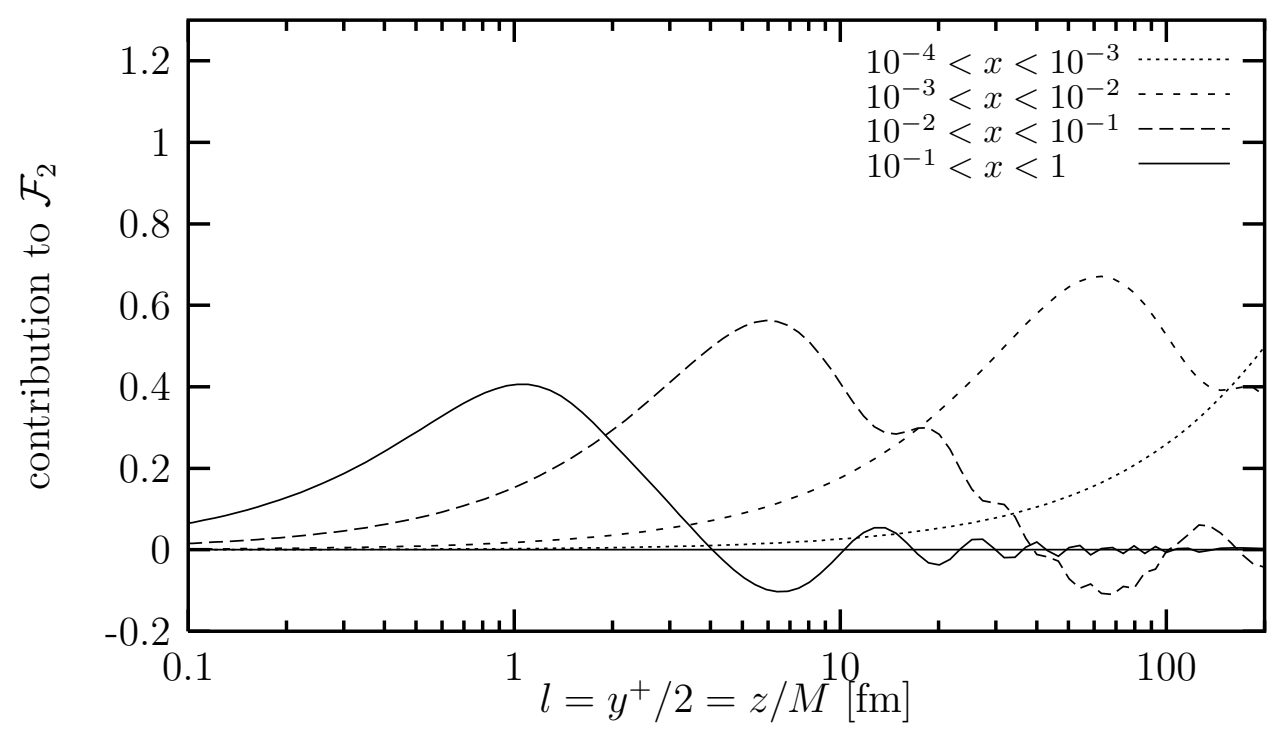

Figure 3: Contributions from different regions in $x$ to the $\mathcal{F}_{2}$ combination of coordinatespace quark and antiquark distributions. The CTEQ4L parametrization of momentumspace distributions, taken at a momentum scale $Q^{2}=4 \mathrm{GeV}^{2}$, has been used. 
At $z<5$ the coordinate-space distributions are determined by average properties of the corresponding momentum-space distribution functions as expressed by their first few moments 12]. For example, the derivative of the $C$-even quark distribution $\mathcal{Q}(z)$ taken at $z=0$ equals the fraction of the nucleon light-cone momentum carried by quarks. The same is true for the gluon distribution $z \mathcal{G}(z)$ (the momentum fractions carried by quarks and by gluons are in fact approximately equal, a well-known experimental fact). At $z>10$ coordinate-space distributions are determined by the small $x$ behavior of the corresponding momentum-space distributions. Assuming, for example, $q(x) \sim x^{\alpha}$ for $x<0.05$ implies $\mathcal{Q}(z) \sim z^{-\alpha-1}$ at $z>10$. Similarly, the small $x$ behavior $g(x) \sim x^{\alpha}$ leads to $z \mathcal{G}(z) \sim z^{-\alpha-1}$ at large $z$. For typical values of $\alpha$ as suggested by Regge phenomenology [13] one obtains $\mathcal{Q}_{v} \sim z^{-0.5}$ while $\mathcal{Q}(z)$ and $z \mathcal{G}(z)$ become constant at large $z$.

The following argument makes it plausible that $z \mathcal{G}(z)$ behaves in a similar way as $\mathcal{Q}(z)$ at large $z$ : In leading order in the strong coupling constant $\alpha_{s}$, gluons enter through $Q^{2}$ evolution in the flavor singlet channel. For the flavor-singlet quark distribution at momentum scale $Q^{2}$ one hast

$$
\begin{aligned}
\frac{\partial \mathcal{Q}\left(z, Q^{2}\right)}{\partial \ln Q^{2}}=-\frac{\alpha_{s}}{2 \pi}\left\{C_{F} \int_{0}^{1} d u K_{\mathcal{Q} \mathcal{Q}}(u) \mathcal{Q}\left(u z, Q^{2}\right)\right. & \\
& \left.+N_{f} \int_{0}^{1} d u K_{\mathcal{Q G}}(u) z \mathcal{G}\left(u z, Q^{2}\right)\right\},
\end{aligned}
$$

where $C_{F}=4 / 3$ and $N_{f}$ is the number of active flavors. The quark-quark and quark-gluon splitting functions read:

$$
\begin{aligned}
K_{\mathcal{Q Q}}(u) & =\frac{1}{2} \delta(1-u)-(1-u)-2 \frac{u}{1-u}-\delta(1-u) \int_{0}^{1} d u^{\prime} \frac{1-u^{\prime}}{u^{\prime}} \\
K_{\mathcal{Q G}}(u) & =-\frac{1}{3}(1-u)\left[2(1-u)^{2}+3 u\right] .
\end{aligned}
$$

We are primarily interested in the region of small Bjorken $x, x<0.01$, which corresponds to large $z \simeq 1 /(2 x)$. Looking at Fig. 20 wee that the integrals in Eq. (14) receive their dominant contributions from the region $u z>5$ or $u>u_{0}=10 x$. In the relevant interval $u_{0}<u \leq 1$ the quark and gluon distributions $\mathcal{Q}(u z)$ and $u z \mathcal{G}(u z)$ are smooth functions which can be replaced approximately by their values at a point $u=\bar{u}$ within the interval. Neglecting corrections from $u<u_{0}$ gives:

$$
\frac{\partial \mathcal{Q}\left(z, Q^{2}\right)}{\partial \ln Q^{2}} \approx-\frac{\alpha_{s}}{2 \pi}\left\{C_{F} \mathcal{Q}\left(\bar{u} z, Q^{2}\right) \int_{u_{0}}^{1} d u K_{\mathcal{Q Q}}(u)+N_{f} \bar{u} z \mathcal{G}\left(\bar{u} z, Q^{2}\right) \int_{u_{0}}^{1} \frac{d u}{u} K_{\mathcal{Q G}}(u)\right\} .
$$

We find indeed that at small values of $x$ or, equivalently, at large $z$ the gluon distribution enters in terms of $z \mathcal{G}(z)$. For $x<0.05$, which corresponds to $u_{0}<0.4$, the integral over the gluon splitting function dominates increasingly over the quark contribution. This reflects the well-established dominance of gluons in the QCD evolution at small $x$ [14].

\footnotetext{
${ }^{2}$ The complete leading order DGLAP evolution equations in coordinate space can be found for example in 10 .
} 
Note that the strong rise of quark and gluon distribution functions at small $x$ and large $Q^{2}$ as observed at HERA leads to an increase of the corresponding coordinate-space distributions at large $z$. In this kinematic region, corrections involving derivatives of the gluon distribution have to be included in Eq. (17).

Finally we illustrate the relevance of large distances in deep inelastic scattering at small $x$ as discussed in the introduction. In Fig. 通 we show contributions to the structure function $F_{2}$ in coordinate space which result from different regions of Bjorken $x$. We confirm indeed that contributions from large distances $\sim 1 /(M x)$ dominate at small $x$.

\section{Nuclear parton distribution functions}

A detailed analysis of nuclear parton distribution functions in momentum space was performed recently in [15] (for earlier investigations see also [16, 17]). In this work a set of nuclear parton distributions at an initial momentum scale $Q_{0}^{2}=2.25 \mathrm{GeV}^{2}$ was determined by using data from deep inelastic lepton-nucleus scattering and Drell-Yan dilepton production in proton-nucleus collisions. Important constraints were imposed by baryon number and momentum conservation. Based on the complete set of these data, a lowest order DGLAP evolution analysis was performed in order to extract separately the quark and gluon content of the nuclear distributions. Good agreement with present experimental data for the ratio of the nuclear to the free nucleon structure function, $F_{2}^{\mathrm{A}} / F_{2}^{\mathrm{N}}$, including its dependence on the momentum transfer $Q^{2}$, was achieved.

We shall consider the following ratios of quark and gluon distribution functions normalized to the number of nucleons in the target:

$$
\begin{aligned}
R_{F_{2}}\left(x, Q^{2}\right) & =\frac{F_{2}^{\mathrm{A}}\left(x, Q^{2}\right)}{F_{2}^{\mathrm{N}}\left(x, Q^{2}\right)}=\frac{\sum_{f} e_{f}^{2}\left[q_{f}^{\mathrm{A}}\left(x, Q^{2}\right)+\bar{q}_{f}^{\mathrm{A}}\left(x, Q^{2}\right)\right]}{\sum_{f} e_{f}^{2}\left[q_{f}^{\mathrm{N}}\left(x, Q^{2}\right)+\bar{q}_{f}^{\mathrm{N}}\left(x, Q^{2}\right)\right]}, \\
R_{v}\left(x, Q^{2}\right) & =\frac{\sum_{f}\left[q_{f}^{\mathrm{A}}\left(x, Q^{2}\right)-\bar{q}_{f}^{\mathrm{A}}\left(x, Q^{2}\right)\right]}{\sum_{f}\left[q_{f}^{N}\left(x, Q^{2}\right)-\bar{q}_{f}^{N}\left(x, Q^{2}\right)\right]}, \\
R_{g}\left(x, Q^{2}\right) & =\frac{g^{\mathrm{A}}\left(x, Q^{2}\right)}{g^{\mathrm{N}}\left(x, Q^{2}\right)} .
\end{aligned}
$$

In Fig. 4 typical results from Ref. [15] are shown for ${ }^{40} \mathrm{Ca}$ and ${ }^{208} \mathrm{~Pb}$ taken at $Q^{2}=4$ $\mathrm{GeV}^{2}$. The ratios shown here and below have been obtained using the GRV-LO parton distributions [18]. It was recently shown in Ref. [19 that the results are insensitive to the choice of the parton distribution set.

The behavior of the structure function ratio $R_{F_{2}}$ and its interpretations are familiar from earlier experimental and theoretical work (see e.g. [2]). At $x<0.1$ the ratio is smaller than one, i.e. there is nuclear shadowing, $F_{2}^{\mathrm{A}}<F_{2}^{\mathrm{N}}$. A small enhancement (" antishadowing") of $F_{2}^{\mathrm{A}}$ is found at $x \sim 0.1$. In the region $0.2<x<0.7$ a significant depletion of $R_{F_{2}}$ (the "EMC effect") can be seen. The strong rise of $R_{F_{2}}$ at $x>0.8$ is caused by Fermi motion. Qualitatively similar effects were obtained for the first time in Ref. [16] for gluon and valence quark distributions. 

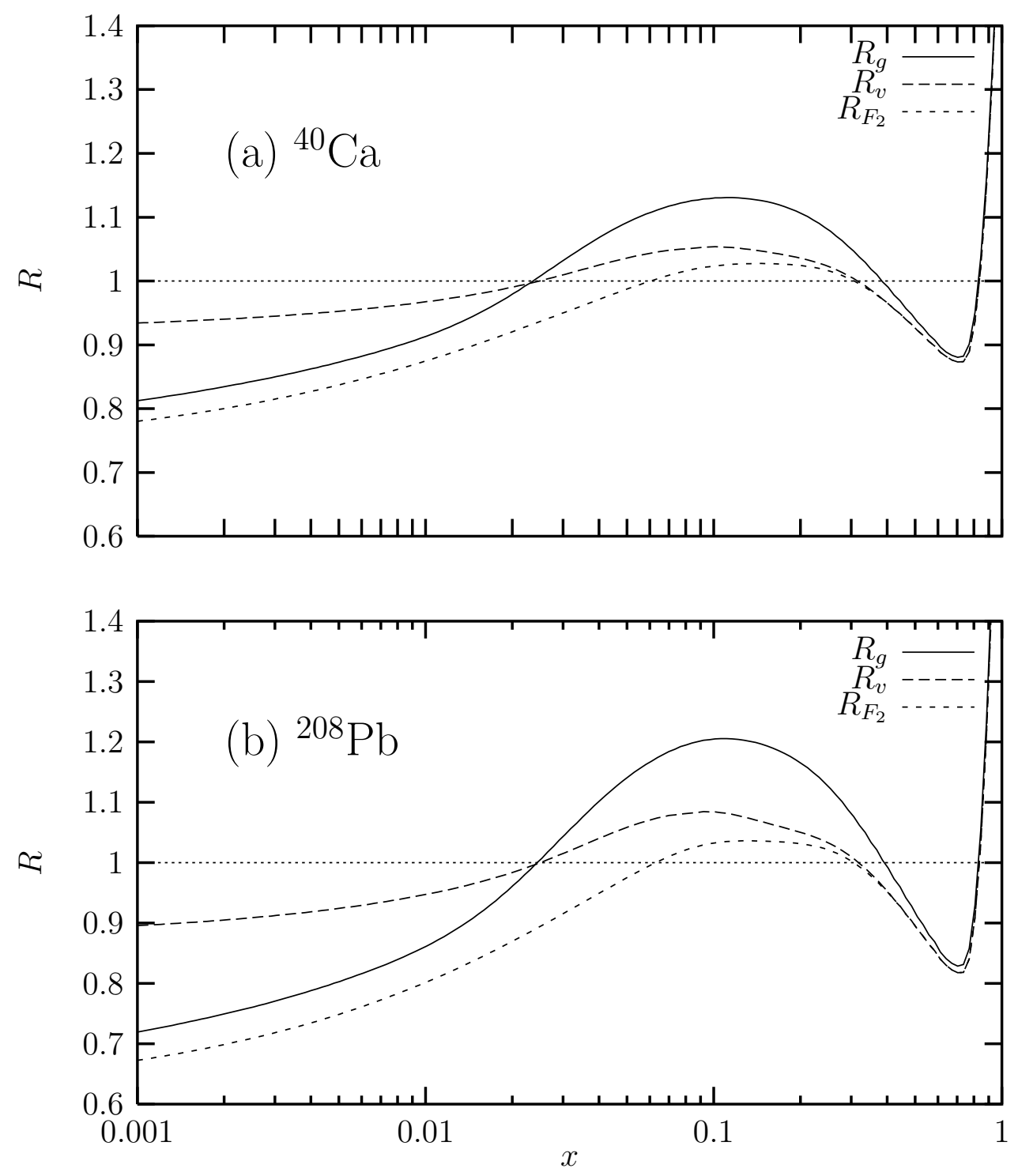

Figure 4: Momentum-space ratios at $Q^{2}=4 \mathrm{GeV}^{2}$ for gluon distributions, valencequark distributions, and the $F_{2}$ structure function in (a) ${ }^{40} \mathrm{Ca}$ and (b) ${ }^{208} \mathrm{~Pb}$ according to Ref. [15]. 
In Ref. [15] it was assumed that at the initial momentum scale $Q_{0}^{2}$ shadowing is of similar size for gluons as for the structure function $F_{2}$, i.e. $R_{g}\left(x, Q_{0}^{2}\right) \approx R_{F_{2}}\left(x, Q_{0}^{2}\right)$ for $x<0.01$. Because of momentum conservation, gluon shadowing at small $x$ implies antishadowing of nuclear gluon distributions at larger $x$. An analysis of the $Q^{2}$ dependence of the structure function ratio $F_{2}^{\mathrm{Sn}} / F_{2}^{\mathrm{C}}$ [20] has shown that gluon antishadowing in $\mathrm{Sn}$ is located in the region $0.03<x<0.4$. In [15] it was assumed that this applies also to other nuclei. Antishadowing was then found to reach its maximum at $x \simeq 0.15$, the maximum being $13 \%$ for ${ }^{40} \mathrm{Ca}$ and $20 \%$ for ${ }^{208} \mathrm{~Pb}$, as shown in Fig. 4 .

The E772 Drell-Yan data show negligible antishadowing of the nuclear quark sea [21]. To account for the small but significant antishadowing of $F_{2}$ at $x \simeq 0.15$, nuclear valence quarks have to be enhanced in this region. Baryon number conservation then implies shadowing at $x<0.1$ also for valence quarks.

Finally let us mention that in Ref. 15 the gluon and sea quark distributions were assumed to show, at $x>0.4$, an "EMC effect" similar to $F_{2}$. Since both distributions are small in this domain, this assumption is however of minor importance for our study.

\subsection{Coordinate-space results}

Using the momentum-space distributions from [15] we have calculated the corresponding ratios for nuclear and nucleon coordinate-space distribution functions:

$$
\begin{aligned}
\mathcal{R}_{F_{2}}\left(z, Q^{2}\right) & =\frac{\int_{0}^{A} \frac{d x}{x} F_{2}^{\mathrm{A}}\left(x, Q^{2}\right) \sin (z x)}{\int_{0}^{1} \frac{d x}{x} F_{2}^{\mathrm{N}}\left(x, Q^{2}\right) \sin (z x)}=\frac{\sum_{f} e_{f}^{2} \mathcal{Q}_{f}^{\mathrm{A}}\left(z, Q^{2}\right)}{\sum_{f} e_{f}^{2} \mathcal{Q}_{f}^{\mathrm{N}}\left(z, Q^{2}\right)} \\
\mathcal{R}_{v}\left(z, Q^{2}\right) & =\frac{\mathcal{Q}_{v}^{\mathrm{A}}\left(z, Q^{2}\right)}{\mathcal{Q}_{v}^{\mathrm{N}}\left(z, Q^{2}\right)}, \\
\mathcal{R}_{\mathcal{G}}\left(z, Q^{2}\right) & =\frac{z \mathcal{G}^{\mathrm{A}}\left(z, Q^{2}\right)}{z \mathcal{G}^{\mathrm{N}}\left(z, Q^{2}\right)} .
\end{aligned}
$$

Results for ${ }^{40} \mathrm{Ca}$ and ${ }^{208} \mathrm{~Pb}$ are presented in Fig. 5.

At large longitudinal distances $l>2 \mathrm{fm}$ a strong depletion of nuclear parton distributions is found. As discussed in Section 3, the asymptotic behavior of coordinate-space distributions at large $l$ is determined by the small $x$ asymptotics of the momentumspace distributions. The coordinate-space ratio at large distances thus corresponds to the nuclear shadowing seen in momentum space at $x<0.1$. Note, however, that at finite distances the coordinate-space distributions correspond to a weighted integral of momentum-space distributions. A given region in momentum space is not simply mapped into a particular finite region in coordinate space. It would, for example, be incorrect to associate the $\mathcal{R}_{g}<1$ region in Fig. 5 exclusively with the $R_{g}<1$ region in Fig. 4 . Instead, the antishadowing region of momentum space also contributes significantly up to $l \lesssim 10$ fm. We shall illustrate the mapping of momentum-space regions into coordinate space in more detail in Section 4.2.

\footnotetext{
${ }^{3}$ Further assumptions, which are not of immediate relevance for our discussion here, are explained in the original paper [15].
} 
Shadowing in deep inelastic scattering in the laboratory frame can be interpreted as being caused by the coherent scattering of hadronic fluctuations of the exchanged virtual photon with several nucleons in the target. This picture is nicely consistent with the observation, in Fig. 5, that the nuclear depletion in coordinate space begins at distances close to the average nucleon-nucleon separation in nuclei, $l \sim 2 \mathrm{fm}$. In momentum space, the Bjorken $x$ value where shadowing begins does not have such a suggestive direct relation to a physical scale.

Another interesting observation is that in coordinate space, shadowing sets in at approximately the same value of $l$ for all sorts of partons. In momentum space, shadowing is found to start at different values of $x$ for different distributions.

At $l \lesssim 1 \mathrm{fm}$, nuclear modifications of parton distributions are small. In this region, deep inelastic scattering proceeds from the individual hadronic constituents in the target nucleus. The intrinsic structure of individual nucleons is evidently not very much affected by nuclear binding.

In the limit $l \rightarrow 0$ the ratio $\mathcal{R}_{F_{2}}$ compares the fractions of target momentum carried by quarks in nuclei and in free nucleons, and $\mathcal{R}_{g}$ compares the momentum fractions carried by gluons. Obviously, $\mathcal{R}_{F_{2}}$ and $\mathcal{R}_{g}$ are correlated because of momentum conservation. For valence quarks, baryon number conservation demands $\mathcal{R}_{v}=1$ for $l \rightarrow 0$.

We find that at small distances $l \leq 1 \mathrm{fm}$ the gluon distribution of bound nucleons is enhanced [16] by a small amount (about 3\% for $\mathrm{Ca}$ ) with respect to free nucleons. The quark distribution is depleted accordingly. This result is, however, consistent with zero within experimental errors (a rigorous error analysis was not performed in Ref. [15], but the error of the total quark momentum integral has been analyzed in Ref. [2]).

In early discussions of the EMC effect [22], the possibility of an increased correlation length of partons in nuclei has been suggested. Within the accuracy of present data, this cannot be verified. Actually it is difficult to give a precise definition of an average correlation length, since the coordinate-space parton distributions are not normalizable and thus cannot be interpreted as parton number densities.

\subsection{Mapping of momentum-space regions into coordinate space}

To disentangle the relationship between nuclear effects of parton distributions in momentum and coordinate space, it is instructive to consider hypothetical momentum-space ratios in which only one of the observed effects (shadowing, antishadowing or the EMC effect) is present. The appropriate Fourier transformations will then show which coordinatespace distances are most closely associated with these momentum-space effects. In the following we consider ${ }^{40} \mathrm{Ca}$, fixing the momentum scale at $Q^{2}=4 \mathrm{GeV}^{2}$ again.

From Fig. 5 we recall that shadowing is clearly the dominant effect in coordinate space. To analyze this further we first choose $R_{v}$ and $R_{g}$ to coincide with the results of [15] in the momentum-space shadowing region and to be equal to 1 outside this region (see Fig. 6a). The resulting coordinate-space ratios are shown in Fig. 6b. The strong depletion of nuclear distributions at large distances $l>10 \mathrm{fm}$ is determined almost completely by 

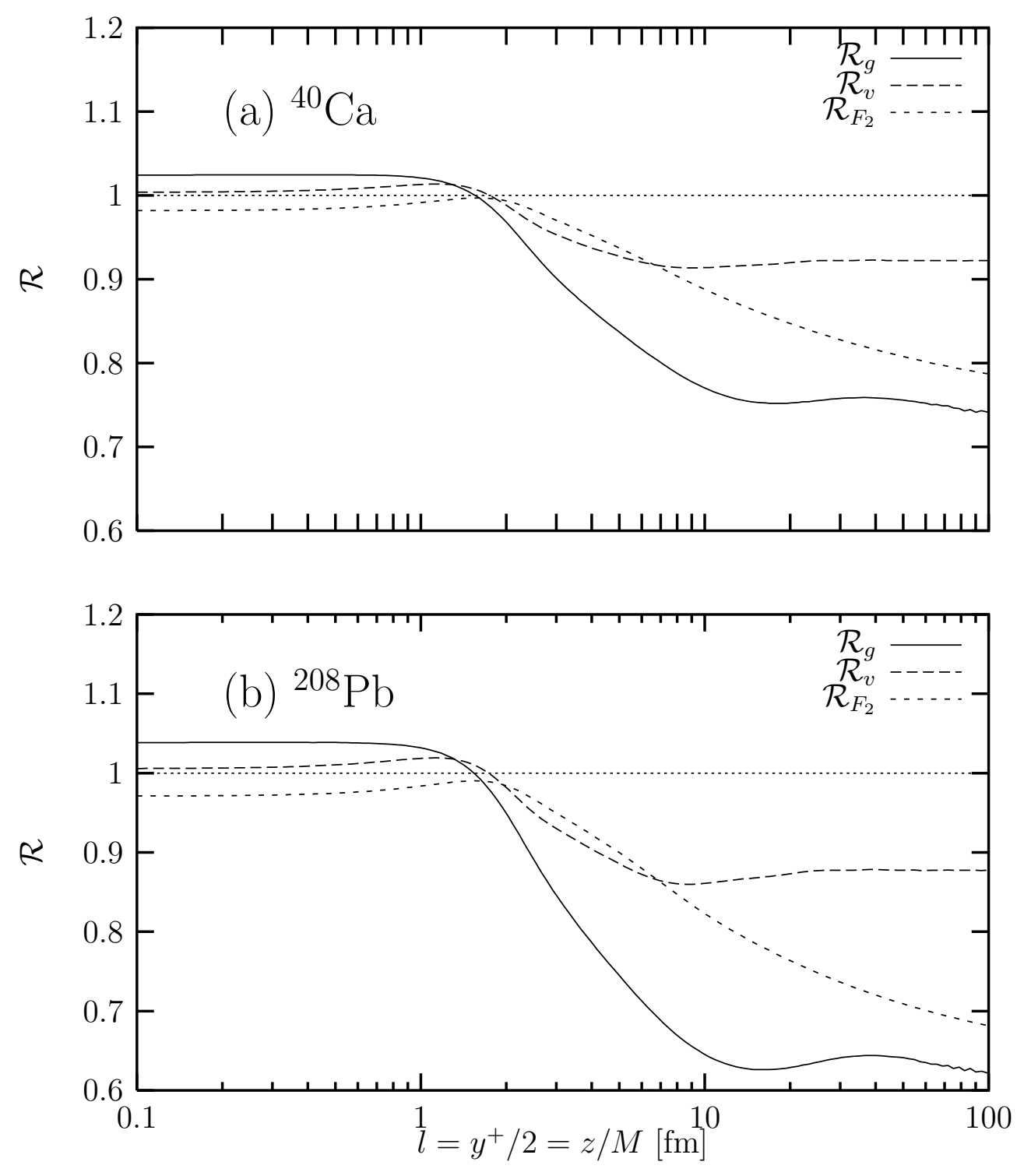

Figure 5: Coordinate-space ratios at $Q^{2}=4 \mathrm{GeV}^{2}$ for gluon distributions, valence-quark distributions, and the $F_{2}$ structure function in (a) ${ }^{40} \mathrm{Ca}$ and (b) ${ }^{208} \mathrm{~Pb}$. 
the momentum-space distribution functions at $x \lesssim 0.1$.

Next we choose $R_{v}$ and $R_{g}$ to coincide with [15] in the momentum-space antishadowing region and to equal 1 elsewhere. By analyzing the corresponding coordinate-space distributions we find that, against naive expectation, the moderate enhancement localized in momentum space around $x \simeq 0.15$ corresponds to effects in coordinate space which spread over a wide range up to $l \sim 10 \mathrm{fm}$.

The binding and Fermi motion effects shown in Fig. 7 have an exaggerated appearance close to $x \sim 1$ simply because the free nucleon distribution function in the denominator of $R_{v}$ decreases rapidly there. These effects turn out to be extremely small when looked at in coordinate space (see Fig. $7 \mathrm{~b}$ ). Clearly, the combined effect of binding and Fermi motion is marginal and leads at most to small fluctuations at the level of $1 \%$.

\section{Summary}

In this paper we have investigated nuclear quark and gluon distributions in coordinate space [5, 6, 24]. Coordinate-space parton distributions are defined as correlation functions involving two quark or gluon fields separated by a light-cone distance $y^{+}$. To study nuclear effects, the corresponding longitudinal distance $l=y^{+} / 2$ has to be compared with typical length scales in nuclei.

The most significant nuclear effects occur at large longitudinal distances. A strong depletion of nuclear parton distributions (shadowing) is found, starting at $l=2 \mathrm{fm}$ which corresponds to the average nucleon-nucleon distance in the bulk of nuclei. In particular we find that shadowing sets in at approximately the same distance $l$ for all sorts of partons. This is different in momentum space, where shadowing starts at different values of Bjorken $x$ for different distributions.

The magnitude of the shadowing effect increases steadily between 2 and $10 \mathrm{fm}$, but there is no trace of geometrical boundary conditions associated with the nuclear radius. Instead, the shadowing effect continues to increase for distances clearly larger than the nuclear radius. This feature is also seen in a number of models for nuclear shadowing (see e.g. 25] and references therein).

At $l \lesssim 1 \mathrm{fm}$, nuclear modifications of parton distributions are very small. The intrinsic structure of individual nucleons is evidently not very much affected by nuclear binding. This observation becomes even more apparent by looking at the combined binding and Fermi motion effects. Their influence on nuclear coordinate-space distributions is at the level of about $1 \%$.

Finally, our analysis does not show evidence for a significant increase of quark or gluon correlation lengths in bound nucleons. 

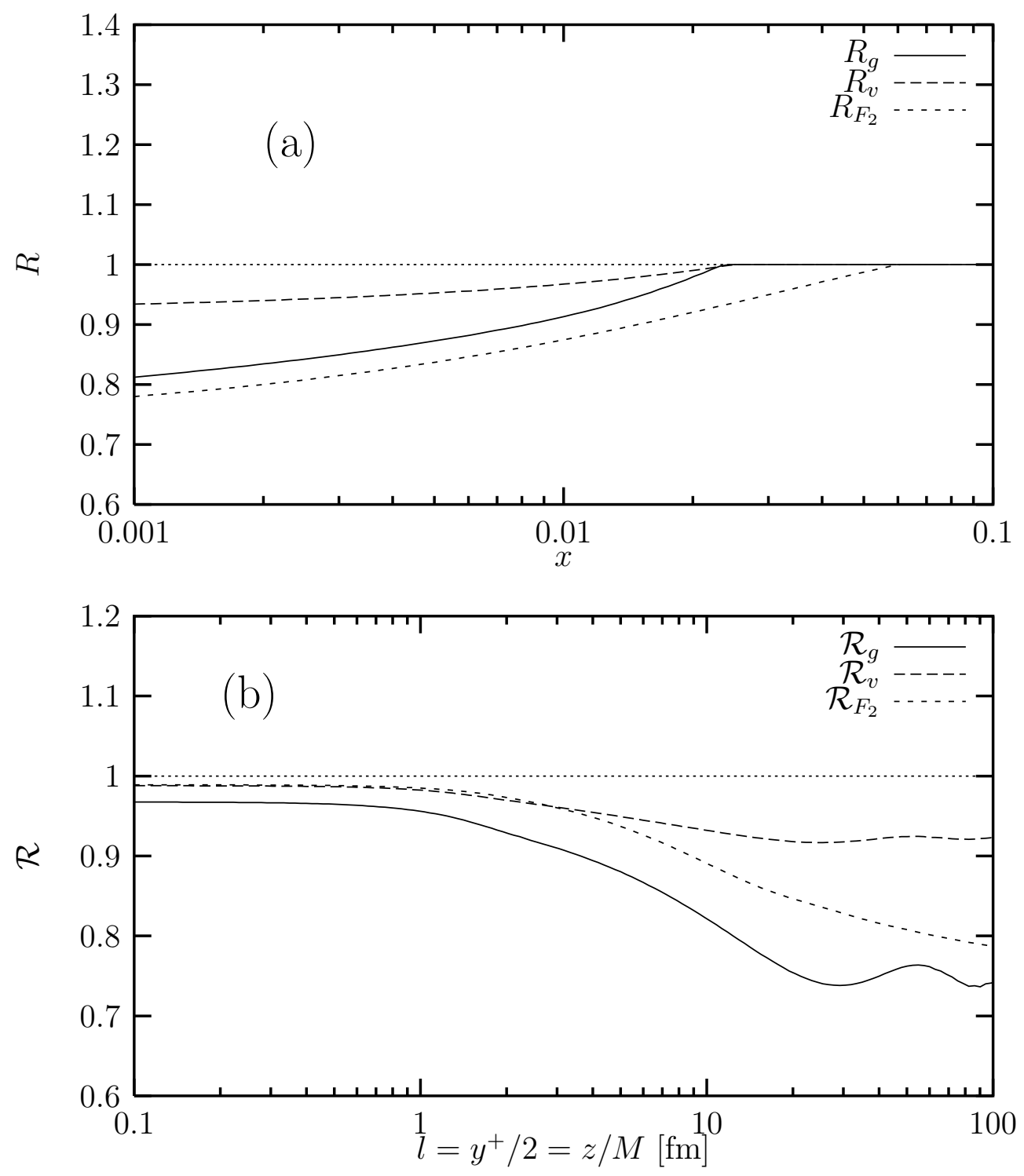

Figure 6: (a) Shadowing effect in ${ }^{40} \mathrm{Ca}$ in momentum space according to Ref. [15]. (b) The coordinate-space ratios for gluon distributions, valence-quark distributions, and the $F_{2}$ structure function in ${ }^{40} \mathrm{Ca}$ which are obtained after setting the momentum-space ratio to one outside the shadowing region. 

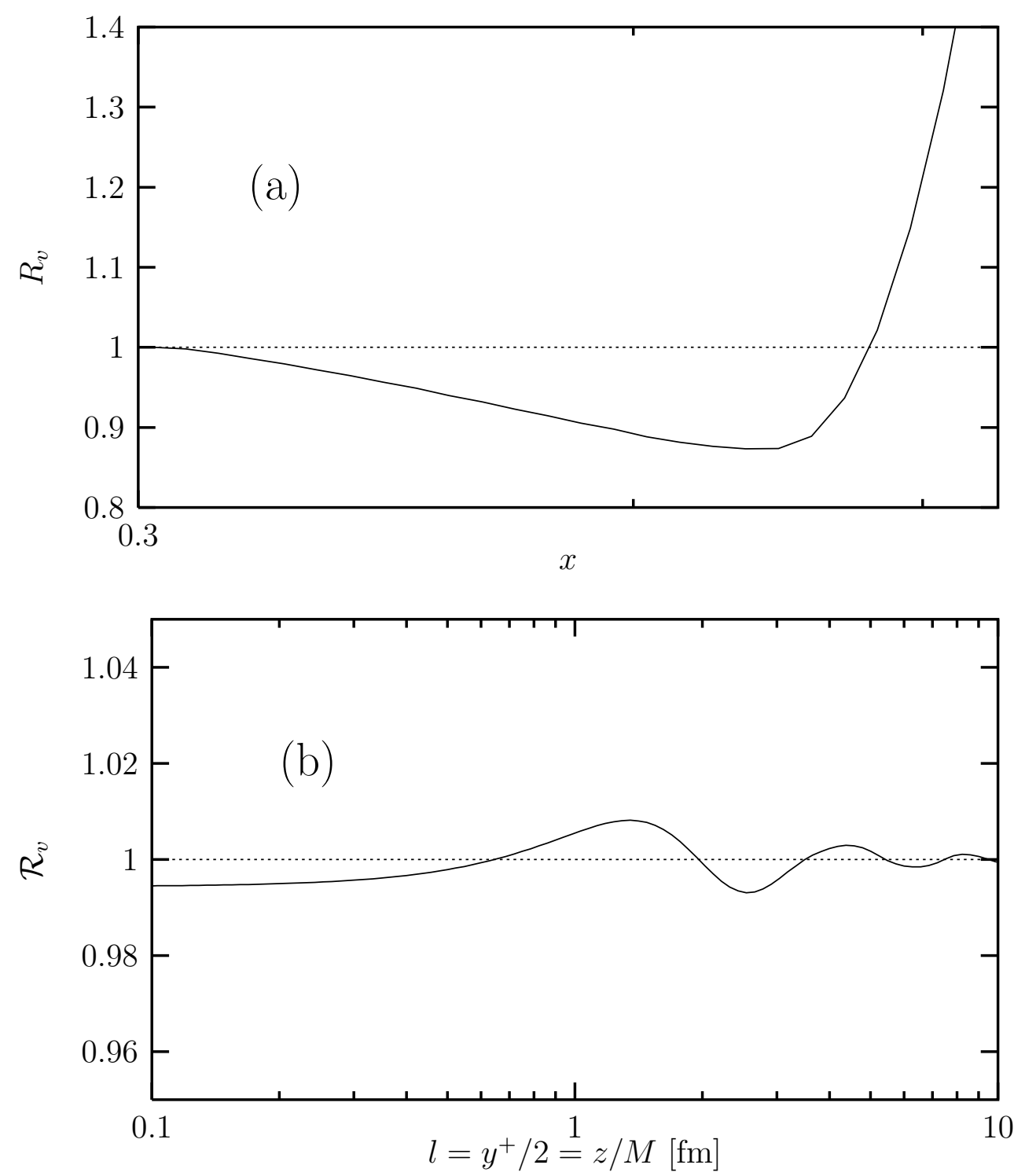

Figure 7: (a) The EMC and Fermi motion effects for valence quarks in ${ }^{40} \mathrm{Ca}$ in momentum space according to Ref. [15]. (b) The coordinate-space ratio for the valence-quark distribution in ${ }^{40} \mathrm{Ca}$ which is obtained after setting the momentum-space ratio to one below the EMC region. 


\section{References}

[1] J. J. Aubert et al. (EMC Collaboration), Phys. Lett. B 105, 322 (1982).

[2] M. Arneodo, Phys. Rep. 240, 301 (1994).

[3] J. C. Collins and D. E. Soper, Nucl. Phys. B 194, 445 (1982).

[4] I. I. Balitsky and V. M. Braun, Nucl. Phys. B 311, 541 (1989).

[5] C. H. Llewellyn Smith, Nucl. Phys. A 434, 35c (1985).

[6] P. Hoyer and M. Vänttinen, Z. Phys. C 74, 113 (1997).

[7] T. Muta, Foundations of Quantum Chromodynamcis, World Scientific, Singapore 1987.

[8] B. L. Ioffe, Phys. Lett. B 90, 123 (1969).

[9] T. H. Bauer, R. D. Spital, D. R. Yennie and F. M. Pipkin, Rev. Mod. Phys. 50, 261 (1978).

[10] V. M. Braun, P. Górnicki and L. Mankiewicz, Phys. Rev. D 51, 6036 (1995).

[11] H. L. Lai et al., Phys. Rev. D 55, 1280 (1997).

[12] L. Mankiewicz and T. Weigl, Phys. Lett. B 380, 134 (1996).

[13] P. D. B. Collins, An Introduction to Regge Theory and High-Energy Physics, Cambridge University Press, Cambridge, 1977.

[14] A. M. Cooper-Sarkar and R. C. E. Devenish and A. De Roeck, Structure functions of the nucleon and their interpretation, 1997, hep-ph/9712301.

[15] K. J. Eskola, V. J. Kolhinen and P. V. Ruuskanen, Scale evolution of nuclear parton distributions, Report No. CERN-TH-97-345, hep-ph/9802350, to appear in Nucl. Phys. B.

[16] L. L. Frankfurt, M. I. Strikman and S. Liuti, Phys. Rev. Lett. 65, 1725 (1990).

[17] K. J. Eskola, Nucl. Phys. B 400, 240 (1993).

[18] M. Glück, E. Reya and A. Vogt, Z. Phys. C 53, 127 (1992).

[19] K. J. Eskola, V. J. Kolhinen and C. A. Salgado, The scale-dependent nuclear effects in parton distributions for practical applications, Report No. JYFL-8-98, hep$\mathrm{ph} / 9807297$.

[20] T. Gousset and H. J. Pirner, Phys. Lett. B 375, 349 (1996). 
[21] D. M. Alde et al., Phys. Rev. Lett. 64, 2479 (1990).

[22] R. L. Jaffe, Phys. Rev. Lett. 50, 228 (1983).

[23] B. L. Ioffe and V. A. Khoze, Hard Processes, North-Holland, Amsterdam, 1984.

[24] L. McLerran and R. Venugopalan, Phys. Lett. B 424, 15 (1998).

[25] B. Badelek and J. Kwiecinski, Nucl. Phys. B 370, 278 (1992); N. N. Nikolaev and V. R. Zoller, Z. Phys. C 56, 623 (1992); W. Melnitchouk and A. W. Thomas, Phys. Lett. B 317, 437 (1993); G. Piller, G. Niesler and W. Weise, Z. Phys. A 358, 407 (1997). 